\section{(C) OPEN ACCESS}

\title{
Genetic screening in sporadic ALS and FTD
}

\author{
Martin R Turner, ${ }^{1}$ Ammar Al-Chalabi, ${ }^{2}$ Adriano Chio, ${ }^{3}$ Orla Hardiman, ${ }^{4}$ \\ Matthew C Kiernan, ${ }^{5}$ Jonathan D Rohrer, ${ }^{6}$ James Rowe, ${ }^{7}$ William Seeley, ${ }^{8}$ Kevin Talbot ${ }^{1}$
}

${ }^{1}$ Nuffield Department of Clinical Neurosciences, Oxford University, Oxford, UK 2Department of Basic and Clinical Neuroscience, King's College London, London, UK ${ }^{3}$ Department of Neuroscience, University of Torino, Torino, Italy ${ }^{4}$ Academic Unit of Neurology, Trinity College Dublin, Dublin, Ireland

${ }^{5}$ Brain and Mind Centre, Royal Prince Alfred Hospital, Sydney, New South Wales, Australia ${ }^{6}$ Dementia Research Centre, UCL Institute of Neurology, London, UK

${ }^{7}$ Department of Clinical Neurosciences, University of Cambridge, Cambridge, UK ${ }^{8}$ Department of Neurology, Memory and Aging Center University of California San Francisco, San Francisco, California, USA

\section{Correspondence to}

Professor Martin R Turner, Nuffield Department of Clinical Neurosciences, West Wing Level 6, John Radcliffe Hospital, Oxford, OX3 9DU, UK; martin. turner@ndcn.ox.ac.uk

Received 7 March 2017 Revised 3 May 2017 Accepted 22 May 2017 Published Online First 22 June 2017

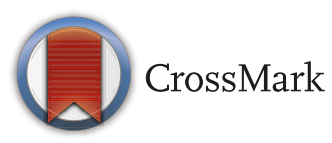

To cite: Turner MR, AlChalabi A, Chio A, et al. J Neurol Neurosurg Psychiatry 2017;88:1042-1044.

\section{ABSTRACT}

The increasing complexity of the genetic landscape in amyotrophic lateral sclerosis (ALS) and frontotemporal dementia (FTD) presents a significant resource and physician training challenge. At least $10 \%$ of those diagnosed with ALS or FTD are known to carry an autosomal dominant genetic mutation. There is no consensus on what constitutes a positive family history, and ascertainment is unreliable for many reasons. However, symptomatic individuals often wish to understand as much as possible about the cause of their disease, and to share this knowledge with their family. While the right of an individual not to know is a key aspect of patient autonomy, and despite the absence of definitive therapy, many newly diagnosed individuals are likely to elect for genetic testing if offered. It is incumbent on the practitioner to ensure that they are adequately informed, counselled and supported in this decision.

\section{INTRODUCTION}

High on the list of questions for those diagnosed with amyotrophic lateral sclerosis (ALS), frontotemporal dementia (FTD) and other neurodegenerative disorders is whether the disease is hereditary. It may be easy for the clinician to overlook the strength of an individual's desire to understand the factors leading to their disease, including genetic causes, even if this does not change specific treatment. If no relevant family history is revealed by a newly diagnosed individual with ALS where good knowledge of the family exists, one study has suggested that their children have almost the same chance of not developing ALS as the general population. ${ }^{1}$ In the context of conveying the news of a terminal diagnosis, the desire to offer some aspect of reassurance will be strong. However, this does not obviate the need to consider the role of genetic testing.

A previous review concluded, based on knowledge current at that time, that testing should not be offered to those with sporadic ALS. ${ }^{2}$ An increased appreciation of the major limitations of family history taken in the clinic, rapid advances in preimplantation screening, increasing availability of commercial genetic testing and the promise of gene-targeted therapy make it timely to reconsider this position. This article considers the arguments for and against offering routine genetic testing to all those with sporadic ALS or FTD.

\section{The genetic landscape of ALS and FTD}

Clinical, pathological and genetic overlap between ALS and FTD is now well established. ${ }^{3}$ Neuronal and glial cytoplasmic inclusions containing the $43 \mathrm{kDa}$ transactive response DNA binding protein, TDP-43, are found in $98 \%$ of all cases of ALS and approximately $50 \%$ of $\mathrm{FTD}^{4}{ }^{4} \mathrm{~A}$ hexanucleotide expansion (G4C2) in intron 1 of the C9orf72 gene is the cause of chromosome 9-associated ${ }^{56}$ and linked ${ }^{7} 8$ pure ALS and FTD (typically the behavioural variant), and mixed ALS-FTD, ${ }^{10}$ with multiple phenotypes seen within the same pedigree. ${ }^{11}$ Based on meta-analysis of international cohorts (mainly Western hemisphere), 5\% of those with ALS are recorded as familial. ${ }^{12}$ In FTD, this number is higher, at approximately $25 \%-30 \%$. Approximately one-third of ALS and FTD cases will harbour a pathogenic C9orf72 expansion. Screening of individuals with ALS or FTD, but without an apparent family history of either, reveals up to $10 \%$ as carriers of the expansion. While there are about 30 genes in which variation has been repeatedly associated with ALS, these account collectively for a much smaller proportion of cases. ${ }^{13}$ Fewer genes have been associated with FTD, but mutations in GRN and MAPT are common causes, although less frequent than C9orf72 expansions. Although both ALS and FTD are typically disorders of middle-to-late life, some genes, for example FUS, have been associated with juvenile forms ${ }^{14}$ (see figure 1).

The clinical distinction between familial and sporadic disease is unreliable

The distinction between familial and sporadic disease can be influenced by ascertainment bias, and there is a growing appreciation of the limitations of family history taking in the clinic, compounded by a lack of a uniform definition of familial disease..$^{15} 16$ Boundaries will also widen significantly with the inclusion of related disorders (including autism, schizophrenia, bipolar disease, multiple sclerosis and Parkinson's disease ${ }^{17}$ ). A reliable family history of cognitive disorders is another challenging area, specifically differentiating Alzheimer's disease from FTD in relatives based on clinical history alone, with a risk of inflating or underestimating the frequency of a positive family history.

A single gene variation may result in different phenotypes, which may reduce ascertainment if the alternative phenotypes are not recognised as relevant. C9orf72 expansions have been associated with diagnoses of dementia (including behavioural variant FTD, primary progressive aphasia and even Alzheimer-type dementia), ataxia, chorea and schizophrenia. Such disorders may well be overlooked by respondents when asked about their family history in the context of an ALS assessment. 


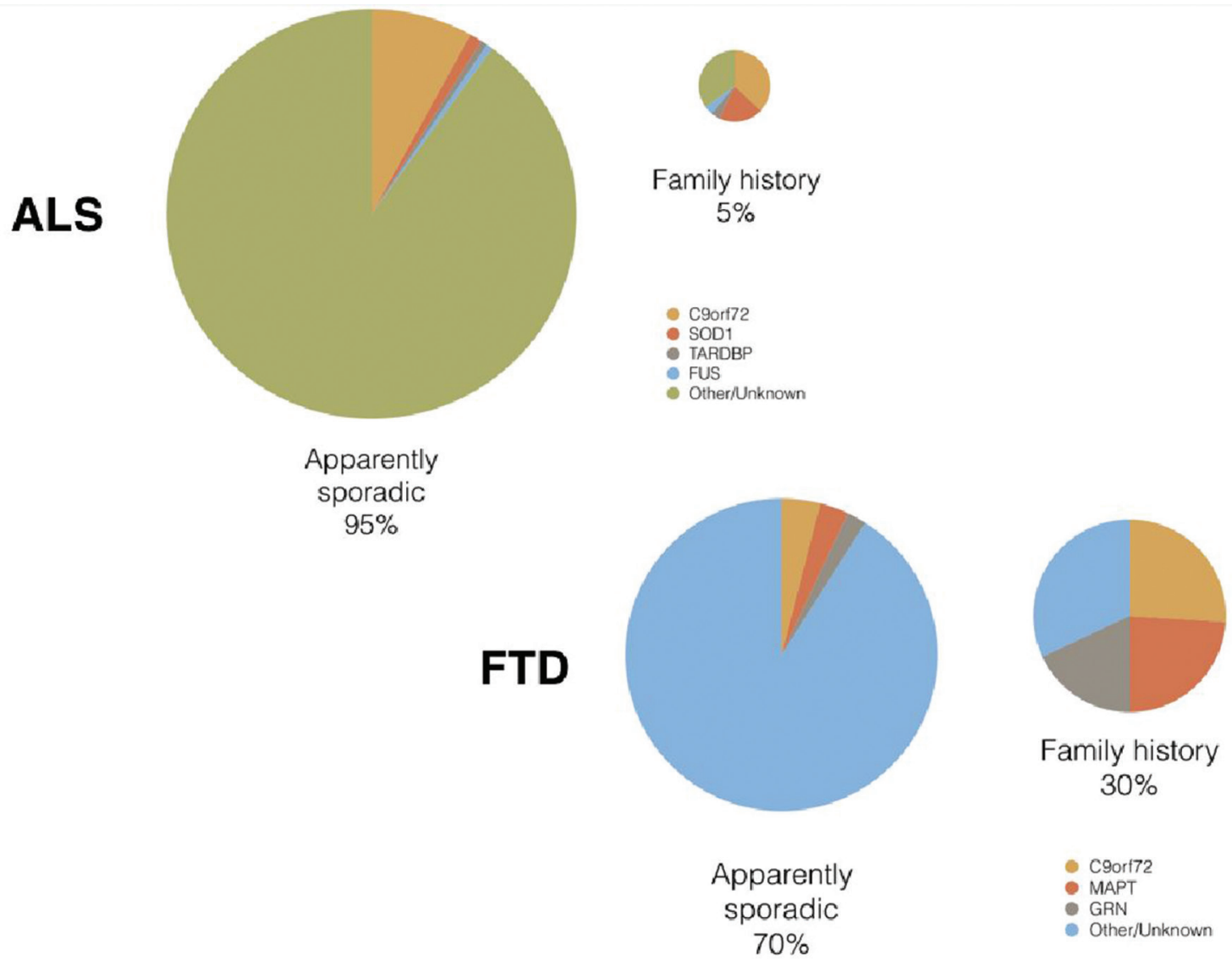

Figure 1 Upper panel: Comparison of proportions of monogenic causes of ALS in those reporting a family history of ALS versus apparently sporadic cases. Lower panel: Comparison of proportions of monogenic causes of FTD in those reporting a family history of dementia versus apparently sporadic cases. ALS, amyotrophic lateral sclerosis; FTD, frontotemporal dementia.

The penetrance of many ALS genes is age-dependent and incomplete, and family size directly influences the probability of an affected relative. ${ }^{18}$

The distinction between familial and sporadic ALS is therefore not clear-cut, and this is underscored by the observation that every established familial ALS gene has also been implicated in sporadic ALS. ${ }^{19}$ Penetrance, the probability of manifesting a phenotype given one is a carrier of the risk genotype, is closely related to the frequency and effect size of disease-associated gene variants. Genotypes associated with high penetrance typically have very large effect sizes and are usually rare. Low penetrance genotypes are usually associated with a small effect. For several ALS genes, including the C9orf72 expansion mutation, penetrance has been reported as high within affected families, 1120 but this is not what is expected from observation of the ratio of familial and sporadic frequencies in the UK, ${ }^{21}$ which predicts an overall penetrance of 38\% (http://alsod.iop.kcl.ac.uk/misc/ penetrance.aspx), nor from the very strong genetic association signal in large studies of people with apparently sporadic ALS. ${ }^{22}$ Incomplete penetrance, and the current lack of a gold standard for how to measure it, complicates decisions of whether to test in the absence of a family history and how to interpret a positive result. A further complexity is the rare occurrence of de novo mutations, which has been described in FUS $S^{14}$ and SOD $1 .{ }^{23}$

\section{The argument against routinely offering C9orf72 testing}

At present, there is no disease-modifying or neuroprotective therapy for C9orf72-related disease, and uncertainty remains over factors influencing penetrance, which may vary between individual families. Consent to test cannot be truly informed if there is insufficient information. Although clinical trials are underway, until an effective therapy is available, a positive test for the C9orf72 expansion in someone with no family history of ALS or FTD has life-changing implications for relatives. There is therefore understandable concern that cannot yet be offset by the prospect of an effective treatment. There will be consequent pressure for consideration of presymptomatic testing, the counselling for which requires high-quality evidence. Individuals might be strongly advised to involve other family members in their decision to undergo testing, but there is a risk that a healthy individual's right not to know about their own risk might be inadvertently breached. Uncertainty is compounded by the difficulty in interpretation of a positive gene test in the absence of a relevant family history, since there will have been many obligate carriers who did not manifest disease or who developed a different but related condition.

\section{The argument for offering C9orf72 testing routinely}

It is now possible for at least $10 \%$ of all those diagnosed with ALS and FTD to understand the cause of their disease, and to share this knowledge with relatives to allow them to make decisions about learning their own and any future children's genetic status. For the symptomatic individual, it can bring a degree of understanding and accommodation to what is otherwise a random and unexplained blow.

For the newly diagnosed individual, a clinician may be tempted to withhold information or defer testing because knowledge is incomplete, or through concern that the individual or their 
family will be further burdened in the absence of a therapy. In many countries, there are no longer healthcare system barriers to accessing personal genetic testing, and the provision of impartial and evidence-based information by experienced clinicians is preferable to individuals seeking information in an unguided, unfiltered way through the internet.

Newly diagnosed individuals may not wish to burden their children with worries over the future, but might equally be keen to offer them the option to consider preimplantation screening, especially in circumstances where it could be undertaken without disclosing the presence of an obligate carrier.

Finally, established international research consortia such as the Presymptomatic Neurodegeneration Initiative and Genetic Frontotemporal Dementia Initiative anticipate exciting new therapeutic options. For example, antisense oligonucleotide therapy against wild-type SOD1 is about to enter its first trials in ALS, and similar therapies for C9orf72 expansions are likely in the next few years. Information about anticipated developments that are directly relevant to known mutations may be a compelling part of the decision-making regarding genetic testing for some newly diagnosed individuals.

The imminent availability of therapy has also been identified as a major factor in changing physician practice in relation to routine testing, ${ }^{23}$ although benefit from genetic therapy in established neurodegenerative disorders remains unproven, and the demonstration of efficacy in disease prevention is likely to be years away.

It is noteworthy that a survey of 167 clinicians from 21 different countries (the majority of whom identified themselves as having a specialist interest in ALS) revealed that more than half would seek genetic testing if they had personally received the diagnosis. ${ }^{24}$ However, it must also be noted that there are practical issues in relation to the clinical expertise needed to provide nuanced and tailored conversations with individuals from diverse backgrounds and to ensuring that resources are equally available to all and understandable across a range of educations and backgrounds. There are also issues in relation to the financial cost to the individual of any testing. Importantly, many neurologists lack the specialised training of clinical geneticists. Familiarity with the complex issues involved and knowledge of who to refer to specialist services is therefore vital for specialists and trainees ${ }^{25}$ to fully realise the benefits of the rapid genetic advances for all those diagnosed with ALS and FTD, and their families.

\section{CONCLUSIONS}

Across the clinical neurosciences, there is increased understanding of the need to provide expert interpretation of freely available scientific knowledge. It is incumbent on the medical profession to recognise patient autonomy and to support decision-making by those who strongly believe that they are maximising options for their own children and wider family, as well as trying to understand their own disease, while also recognising the right not to know one's genetic status. ${ }^{26}$ Either way, the practice of refraining from any discussion of genetic testing should now be challenged as an unnecessary limitation to the provision of best care in ALS and FTD.

Contributors MRT conceived and drafted the manuscript, and is guarantor for the content. AAC, AC, OH, MCK, JDR, JR, WS and KT edited the manuscript.

Competing interests None declared.
Provenance and peer review Not commissioned; externally peer reviewed.

Open Access This is an Open Access article distributed in accordance with the terms of the Creative Commons Attribution (CC BY 4.0) license, which permits others to distribute, remix, adapt and build upon this work, for commercial use, provided the original work is properly cited. See: http://creativecommons.org/licenses/by/4.0/

(C) Article author(s) (or their employer(s) unless otherwise stated in the text of the article) 2017. All rights reserved. No commercial use is permitted unless otherwise expressly granted.

\section{REFERENCES}

1 Hanby MF, Scott KM, Scotton W, et al. The risk to relatives of patients with sporadic amyotrophic lateral sclerosis. Brain 2011:134(Pt 12):3454-7.

2 Chiò A, Battistini S, Calvo A, et al.Genetic counselling in ALS: facts, uncertainties and clinical suggestions. J Neurol Neurosurg Psychiatry 2014;85:478-85.

3 Burrell JR, Halliday GM, Kril JJ, et al. The frontotemporal dementia-motor neuron disease continuum. Lancet 2016:388:919-31.

4 Neumann M, Sampathu DM, Kwong LK, et al. Ubiquitinated TDP-43 in frontotemporal lobar degeneration and amyotrophic lateral sclerosis. Science 2006;314:130-3.

5 van Es MA, Veldink JH, Saris CG, et al. Genome-wide association study identifies 19p13.3 (UNC13A) and 9p21.2 as susceptibility loci for sporadic amyotrophic lateral sclerosis. Nat Genet 2009:41:1083-7.

6 Shatunov A, Mok K, Newhouse S, et al. Chromosome 9p21 in sporadic amyotrophic lateral sclerosis in the UK and seven other countries: a genome-wide association study. Lancet Neurol 2010;9:986-94.

7 Morita M, Al-Chalabi A, Andersen PM, et al. A locus on chromosome 9p confers susceptibility to ALS and frontotemporal dementia. Neurology 2006;66:839-44.

8 Vance C, Al-Chalabi A, Ruddy D, et al. Familial amyotrophic lateral sclerosis with frontotemporal dementia is linked to a locus on chromosome 9p13.2-21.3. Brain 2006;129(Pt 4):868-76

9 Renton AE, Majounie E, Waite A, et al. ITALSGEN Consortium. A hexanucleotide repeat expansion in C9ORF72 is the cause of chromosome 9p21-linked ALS-FTD. Neuron 2011;72:257-68.

10 DeJesus-Hernandez M, Mackenzie IR, Boeve BF, et al. Expanded GGGGCC hexanucleotide repeat in noncoding region of C9ORF72 causes chromosome 9p-linked FTD and ALS. Neuron 2011;72:245-56.

11 Majounie E, Renton AE, Mok K, et al. Frequency of the C9orf72 hexanucleotide repeat expansion in patients with amyotrophic lateral sclerosis and frontotemporal dementia: a cross-sectional study. Lancet Neurol 2012;11:323-30.

12 Byrne S, Walsh C, Lynch C, et al. Rate of familial amyotrophic lateral sclerosis: a systematic review and meta-analysis. J Neurol Neurosurg Psychiatry 2011;82:623-7.

13 Al-Chalabi A, van den Berg LH, Veldink J. Gene discovery in amyotrophic lateral sclerosis: implications for clinical management. Nat Rev Neurol 2017;13:96-104.

14 Bäumer D, Hilton D, Paine SM, et al. Juvenile ALS with basophilic inclusions is a FUS proteinopathy with FUS mutations. Neurology 2010;75:611-8.

15 Byrne $\mathrm{S}$, Elamin $\mathrm{M}$, Bede $\mathrm{P}$, et al. Absence of consensus in diagnostic criteria for familial neurodegenerative diseases. J Neurol Neurosurg Psychiatry 2012;83:365-7.

16 Byrne S, Bede P, Elamin M, et al. Proposed criteria for familial amyotrophic lateral sclerosis. Amyotroph Lateral Scler 2011;12:157-9.

17 Byrne S, Heverin M, Elamin M, et al. Aggregation of neurologic and neuropsychiatric disease in amyotrophic lateral sclerosis kindreds: a population-based case-control cohort study of familial and sporadic amyotrophic lateral sclerosis. Ann Neurol 2013;74:699-708.

18 Al-Chalabi A, Lewis CM. Modelling the effects of penetrance and family size on rates of sporadic and familial disease. Hum Hered 2011;71:281-8.

19 Talbot K. Familial versus sporadic amyotrophic lateral sclerosis--a false dichotomy? Brain 2011:134(Pt 12):3429-34.

20 Van Langenhove T, van der Zee J, Gijselinck I, et al. Distinct clinical characteristics of C9orf72 expansion carriers compared with GRN, MAPT, and nonmutation carriers in a Flanders-Belgian FTLD cohort. JAMA Neurol 2013;70:365-73.

21 Barbeito LH, Pehar M, Cassina P, et al. A role for astrocytes in motor neuron loss in amyotrophic lateral sclerosis. Brain Res Brain Res Rev 2004;47(1-3):263-74.

22 van Rheenen W, Shatunov A, Dekker AM, et al. Genome-wide association analyses identify new risk variants and the genetic architecture of amyotrophic lateral sclerosis. Nat Genet 2016:48:1043-8.

23 Arthur KC, Doyle C, Chiò A, et al. Use of Genetic Testing in Amyotrophic Lateral Sclerosis by Neurologists. JAMA Neurol 2017;74:125-6.

24 Vajda A, McLaughlin RL, Heverin M, et al. Genetic testing in ALS: A survey of current practices. Neurology 2017;88:991-9.

25 Roggenbuck J, Quick A, Kolb SJ. Genetic testing and genetic counseling for amyotrophic lateral sclerosis: an update for clinicians. Genet Med 2017;19:267-74.

26 Andorno R. The right not to know: an autonomy based approach. J Med Ethics 2004:30:435-9.

27 Alexander MD, Traynor BJ, Miller N, et al. "True" sporadic ALS associated with a novel SOD-1 mutation. Ann Neurol 2002:52:680-3. 